\title{
Discussion and analysis of environmentally friendly refrigerant R404A as a substitute for R22 with different circuit number
}

\author{
Xue Wang ${ }^{1, *}$, Yu Zhang ${ }^{1}$, and Ruoyu Wang ${ }^{1}$ \\ ${ }^{1}$ Shandong Huayu University of Technology, College of Energy and Environmental Engineering, 253034, Dezhou, Shandong, China
}

\begin{abstract}
The refrigerant R22 has great damage to the ozone layer. R404A is currently the mainstream environmentally friendly refrigerant approved and recommended by most countries in the world, and has zero damage to the ozone layer. Based on this, the performance of heat exchangers of R22 and R404A with different inlet qualities with the circuit number were simulated and compared by EVAP-COND software. The results show that the capacity of the evaporator with R404A was greater than that with R22. Moreover, the difference in the capacity of the evaporator with R410A and R22 drops first, and then it rises. The flow path with the number of circuits 2 had the largest heat exchange rate. The heat exchange rates of R22 and R404A are $4.40 \mathrm{~kW}$ and $4.50 \mathrm{~kW}$, respectively. The heat exchange amount of R404A was larger than that of R22, and the difference in heat exchange amount decreases first and then increases with the increase of branches, and the maximum difference was $4.41 \%$. R404A can be used as a substitute for R22 and had a higher heat exchange rate. It is more environmentally friendly and saves energy.
\end{abstract}

\section{Introduction}

Heat exchanger is a kind of heat exchange equipment widely used in industrial fields such as refrigeration, air conditioning, and chemical industry.The finned tube heat exchanger was a heat exchange element that increases the heat transfer area on the outside of the tube to improve the heat transfer capability of the air side. It has the advantages of low manufacturing cost, simple structure and strong applicability. With the development of industrial production, higher requirements are put forward for the performance of heat exchangers. In order to further strengthen the heat transfer capacity of the finned tube, it was necessary to conduct an in-depth study on the fin structure and arrangement. The number of loops had a particularly significant influence on the heat exchanger.

Many researchers have done various researches. Huang Dong et al. simulated and compared the performance of the heat exchanger with the number of circuits when R22 and R410A refrigerants were used in the indoor heat exchanger. ${ }^{[1]}$ Wang Yunli et al. studied the flow of the four-row fin-tube indoor heat exchanger for evaporation and condensation when the air inlet state and air volume, the structural parameters of the heat exchanger, and the refrigerant mass flow ware constant. The influence law of heat transfer performance. ${ }^{[2]}$ Liu Jinping et al. studied the effect of branch number and tube row number on heat transfer oefficient and flow characteristic under the condition of the same geometry size of condenser, air inlet temperature and flow rate was analyzed. The results show that pressure drop decreases as branch numberincreases. ${ }^{[3]}$ Yang Ting et al. used computer simulation technology under the conditions of the same geometrical structure and the same air inlet state and flow rate of the air-conditioning finned tube condenser. The influence of the number of loops and the number of tube rows on the heat transfer and flow characteristics of the finned tube condenser was studied. ${ }^{[4]}$

Previous research mainly focused on R22 working fluid. R404A is one of the main alternative working fluids of R22. It is necessary to study the difference between the number of branches on the R22 and R404A condensation and evaporation dual-use phase change heat exchangers, laying a foundation for R404A to replace R22. Theoretical basis. The refrigerant R404A complies with the standards of the US Environmental Protection Organization EPA, SNAP and UL, and complies with the A1 safety level category of the American Society of Heating, Refrigeration and Air-Conditioning Engineers (this is the highest level and harmless to humans). The fin spacing is very small, and it is more difficult to measure the gasliquid two-phase flow. It is difficult and the workload to conduct synchronous experimental research on the local characteristics of the heat transfer of the fin-tube heat exchanger from both sides of the air and refrigerant. Simulation can better obtain the local characteristic parameters of the heat exchanger. As a result, this article intends to comprehensively improve the efficiency of heat pump air conditioning cooling and heating, and to study the feasibility of R404A as a substitute for R22. In this paper, for R22 and R404A refrigerants, simulation software was used to simulate the fin-tube heat exchangers with different numbers of circuits, and the heat transfer of the heat exchangers under different inlet

\footnotetext{
* Corresponding author: tlwx1994@163.com
} 
qualities of the refrigerants was analyzed to explore the number of circuits exchange.

\section{Simulation procedure and Restriction}

\subsection{Simulation procedure}

This article adopts the software EVAP-COND designed by Domanski of NIST in the United States. It had undergone more than ten years of development and improvement. It had a strong representative in the numerical simulation of finned tube heat exchangers and had a wide range of international influences. EVAPCOND adopted a tube-by-tube calculation scheme, that is,

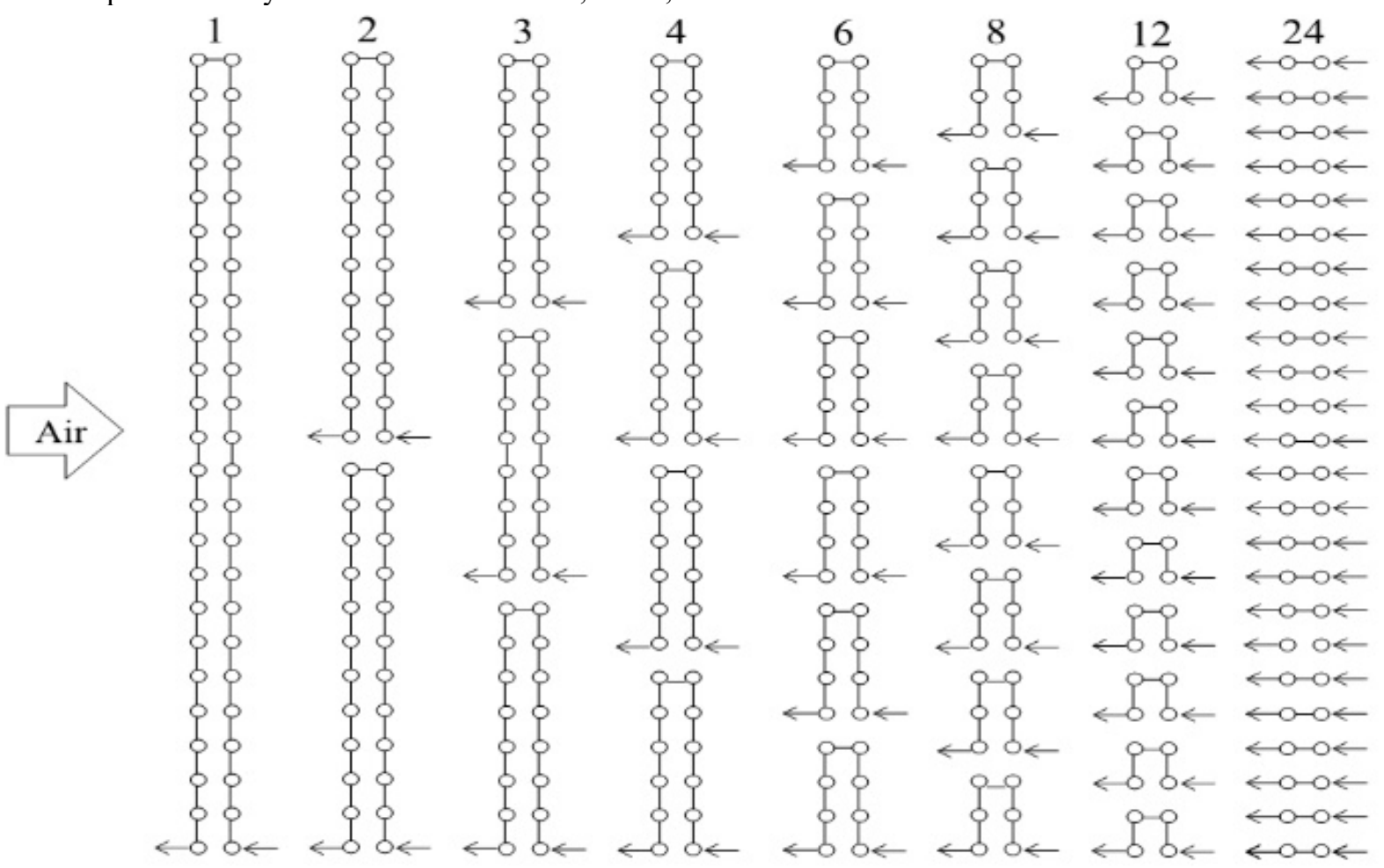

Figure 1. Refrigerant Circuitry.

This paper was studied the difference between the number of circuits on the performance of R22 and R404A refrigerants in the heat pump evaporator, and laid a theoretical foundation for R404A to replace R22. The circuit layout of the refrigerant and the air flow direction were shown in Figure 1. The number of circuits was 1, 2, $3,4,6,8,12$, and 24 respectively. Explore the influence of the number of circuits on the performance of the heat exchanger.

The outlet pressure and temperature of the heat exchanger will not only affect the flow rate of the compressor, but also reflect the evaporation temperature and the size of the superheated section in the heat exchanger. Therefore, the refrigerant outlet state of the heat exchanger was taken as the simulated operating conditions, as shown in Table 1 . The inlet quality reflects the state that the refrigerant enters the evaporator after being throttled by the throttling part. The evaporator mainly transforms heat through the evaporation phase of each heat exchange tube was used as an independent calculation unit. The state and flow of refrigerant and air need to be input separately. For multi-branch arrangement, it was necessary to calculate each branch one by one. ${ }^{[5]}$ In a branch, the calculation was carried out according to the sequence of piping layout and refrigerant flow. The exit parameter of the refrigerant in the former tube was the population parameter of the latter tube. For the bifurcation in the branch, first calculate one of the branches to the exit, and then back to the bifurcation to calculate the other branches.

\subsection{Restriction}

the refrigerant. The greater the dryness, the more flashed gas, and the evaporator can be used. The less effective refrigerant amount, the worse the heat transfer capacity, the lower the dryness, the better the heat transfer capacity.

Table1. Simulation conditions.

\begin{tabular}{|c|c|c|c|}
\hline \multicolumn{4}{|c|}{ Refrigerant } \\
\hline $\begin{array}{c}\text { Inlet } \\
\text { quality }\end{array}$ & \multicolumn{2}{|c|}{$\begin{array}{c}\text { Outlet } \\
\text { saturation } \\
\text { temperature/ } \\
{ }^{\circ} \mathrm{C}\end{array}$} & $\begin{array}{c}\text { Inlet } \\
\text { saturation } \\
\text { temperature/ } \\
{ }^{\circ} \mathrm{C}\end{array}$ \\
\hline $0.1-0.3$ & \multicolumn{2}{|c|}{5} & 5 \\
\hline \multicolumn{4}{|c|}{ Air inlet } \\
\hline \multicolumn{2}{|c|}{$\begin{array}{c}\text { Dry bulb } \\
\text { temperature } /{ }^{\circ} \mathrm{C}\end{array}$} & \multicolumn{2}{|c|}{ Relative humidity $/ \%$} \\
\hline \multicolumn{2}{|c|}{27} & \multicolumn{2}{|r|}{50} \\
\hline
\end{tabular}


The dry and wet bulb temperatures of the inlet air adopt the data of the standard refrigeration conditions in the national standard. In order to make the research results reference and comparative, the structure parameters of the heat exchanger were the same as those in the literature ${ }^{[6]}$, as shown in Table 2.

Table2. Parameters of evaporator.

\begin{tabular}{|c|c|}
\hline Parameters & Number \\
\hline Tube length/mm & 400 \\
\hline Tube size/mm & $9.52 \times 0.35$ \\
\hline Pipe spacing/mm & 25.4 \\
\hline Row spacing/mm & 22 \\
\hline Number of tubes & $3 \times 24$ \\
\hline Fin thickness/mm & 0.11 \\
\hline Fin pitch/mm & 1.5 \\
\hline Air flow $/ \mathrm{m}^{2} / \mathrm{min}$ & 25 \\
\hline
\end{tabular}

\section{Simulation results and analysis}

\subsection{Simulation results of R22 and R404A}

In this simulation, the outlet saturation temperature was $5^{\circ} \mathrm{C}$, and the dryness of the refrigerant was variable. Figures 2 and 3 showed the changes in the heat exchange capacity of different circuit numbers when the inlet quality of different refrigerants R22 and R404A changed.

It can be seen from Figure 2 that the heat exchange amount of R22 first rised and then decreased as the number of circuits increased. When the number of circuits was 2 , the heat exchange was the largest, and when the number of circuits was 24 , the heat exchange was the smallest. And the curve with loop number 1 intersected with the curve with loop number 6 . The inlet quality of the refrigerant had a greater impact on the 24 branches and less on the heat exchangers of other circuits. When the number of circuits were 2, 3, 4 and 6 , the heat exchange amount increased as the inlet quality of the refrigerant increased. There was only 1 circuit and the heat exchange amount decreased with the increase in the inlet quality. The number of circuits 8,12 , and 24 , the change in heat exchange was not monotonous.

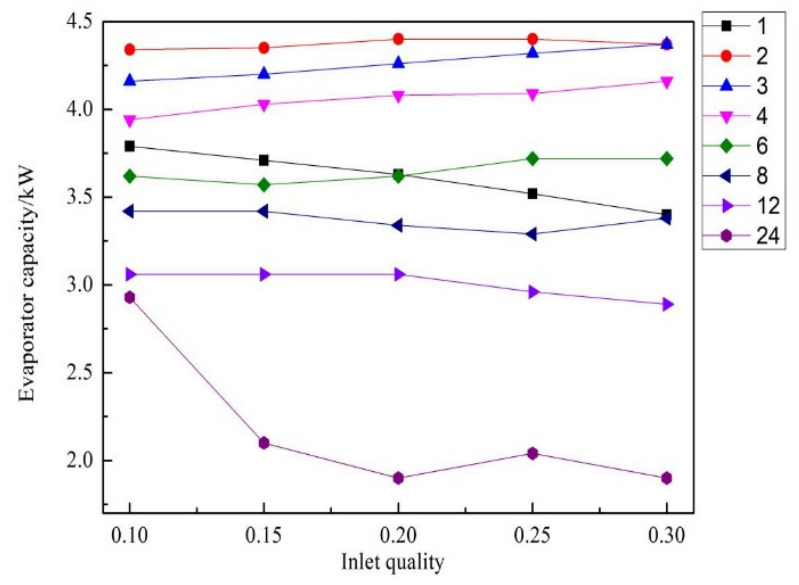

Figure 2. R22 heat exchange.

It can be seen from Fig. 3 that the heat exchange rate of R404A varies with the number of circuits in the same trend as R22, it rised first and then falled. Inlet quality had a small effect on heat transfer, with an overall upward trend, but the flow path with the number of loops shown a downward trend, and the changes in the 12 and 24 branches were irregular.

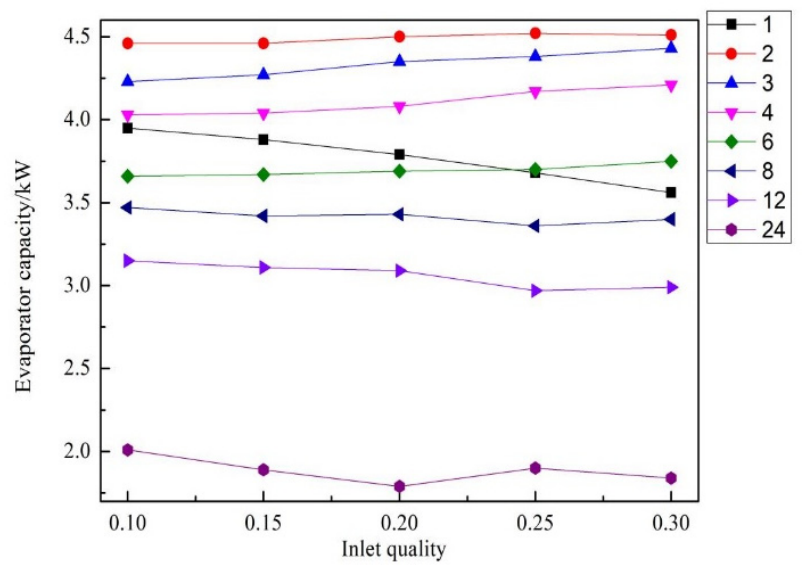

Figure 3. R404A heat exchange.

The Evaporator capacity of all methods increased and then decreased as the dryness increased. The main reason was that the total heat transfer coefficient first increased and then decreased with the change of inlet quality. In the first stage, the disturbance between the bubble and the fluid and between the bubble and the bubble generated by the boiling will intensify the heat transfer effect. Immediately, the total heat transfer coefficient decreased slightly. This was due to the fact that as the refrigerant continues to evaporate during the heat exchange process, the number of bubbles increased, and many small bubbles gradually aggregate to form a block or even a film, which inhibits the refrigerant and the tube wall. Heat transfer. Finally, in the heat exchange process, the liquid refrigerant in the pipe enters the lack of liquid, and the phenomenon of partial evaporation occurs. At the same time, the moisture desorption coefficient on the air side was reduced, and the heat exchange effect on the inner and 
outer sides was affected, resulting in a sharp decrease in the coefficient. ${ }^{[7]}$

\subsection{Comparison results of R22 and R404A}

When used as an evaporator, the difference in heat exchange between R410A and R22 was affected by the heat transfer temperature difference and the heat transfer coefficient, and the heat transfer temperature difference is determined by the inlet temperature of the refrigerant and the outlet temperature of the air. The outlet state of the evaporator is certain. ${ }^{[8]}$

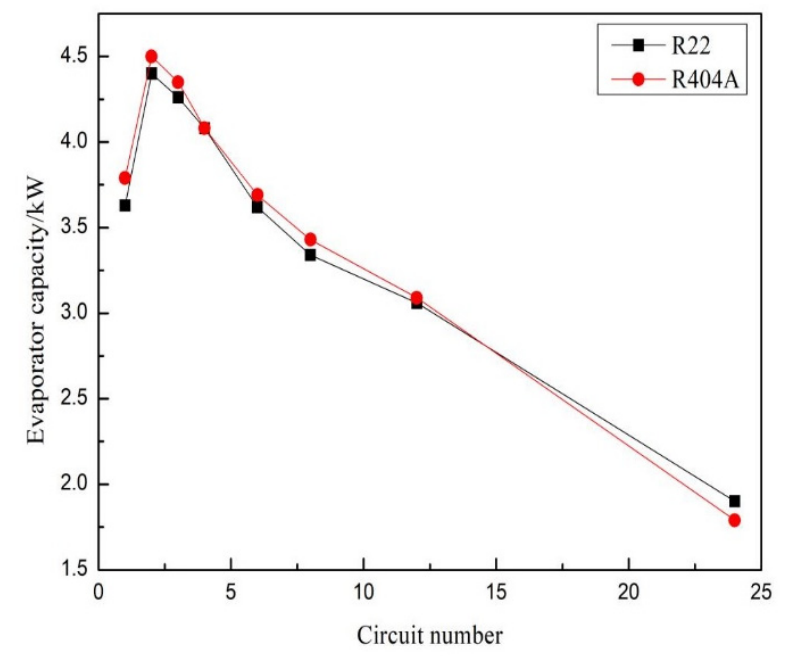

Figure 4. Comparison of R22 and R404A.

In this simulation, the inlet quality value was 0.2 , which compares the heat exchange rate of R22 and R404A. It can be seen from the figure that the changes of the two working fluids first increased and then decreased with the increase of the number of circuits, and the difference in heat exchange first decreases and then increases with the increase of the number of circuits. And the heat transfer capacity of R404A was basically higher than that of R22. When the number of circuits was 1 , the heat exchange amount of R404A was $4.41 \%$ higher than that of R22. When the number of circuits was 2, 3, 6, and 8 , the heat exchange amount of R404A was about $2 \%$ higher than that of R22. When the number of circuits was 4 , they The heat exchange was equal. When the number of circuits was 24 , the heat transfer capacity of R22 was $4.33 \%$ higher than that of R404A.

\section{Conclusion}

This paper compares the performance of the evaporator with the number of loops when the refrigerant is R22 and R404A under the same conditions of the air inlet state and flow, the geometrical structure of the heat exchanger, and the pipe arrangement. The conclusions are as follows:

(1) The heat exchange amount first increases and then decreases with the increase in the number of circuits. The flow path with the number of circuits 2 has the largest heat exchange amount. The heat exchange amounts of R22 and $\mathrm{R} 404 \mathrm{~A}$ are $4.40 \mathrm{~kW}$ and $4.50 \mathrm{~kW}$, respectively.
(2) Inlet quality has less influence on heat exchange. When the inlet quality value is 0.2 , the heat exchange rate of $\mathrm{R} 404 \mathrm{~A}$ is about $2 \%$ higher than that of R22 in most flow paths. The difference of their heat exchange first decreases and then increases with the increase of the number of circuits.

(3) The heat exchange amount of R404A is larger than that of R22, with a maximum difference of $4.41 \%$. It can be seen that R404A can not only be used as a substitute for R22, but also has better performance than R22.

\section{Acknowledgement}

This work was supported by Shandong Huayu University of Technology and Dezhou's Key Laboratory of Highefficiency Heat Pump Air Conditioning Equipment and System Energy Saving Technology (No.26).

\section{References}

1. Huang Dong, Li Quanxu. Performance Comparison of Evaporator with Circuit Number Change in Heat Pump Using R22 and R410A. Journal of Xi'an jiaotong university. J. 43 (2009)

2. Wang Yunli, Yang Bing, Li Ming. Influence of circuit number on performance of R410A indoor heat exchanger. Proceedings of the 6th China Refrigeration and Air Conditioning Industry Information Conference. C. (2012)

3. Liu Jinping, Zheng Qingling. Numerical Simulation of Performance of Fin-and-tube Condenser with Different Structural Arrangement in Air-conditioning Systemand. Refrigeration. J. 28 (2009)

4. Yang Ting. Experiment study and theoretical analysis on the performance of fin packed condensers. Shanghai Jiao Tong University. D. (2014)

5. $\mathrm{Fu}$ Yongjun. The Application of EVAP-COND in Rotor Compressor Matching. Journal of Shunde Polytechnic. J. (2016)

6. Wang Qiang, Liu Yanlong, Liu Zuyi, et al. An Optimized Design and Experimental Research on Finned-tube Evaporator with Nonuniform Air Distribution. Journal of Refrigeration. J. 37 (2016)

7. Li Xia, Weng Wenbing, Mo Pengcheng, et al. Study on heat transfer performance of fin-tube heat exchanger with different section dryness. China Water Transport. J. (2019)

8. Gao Yuping, Shao Shuangquan, Si Chunqiang, et al. Heat Transfer and Pressure Drop Characteristics of R717 Flow Boiling inside a Horizontal Small Diameter Plain Tube. Journal of Refrigeration. J. (2018) 